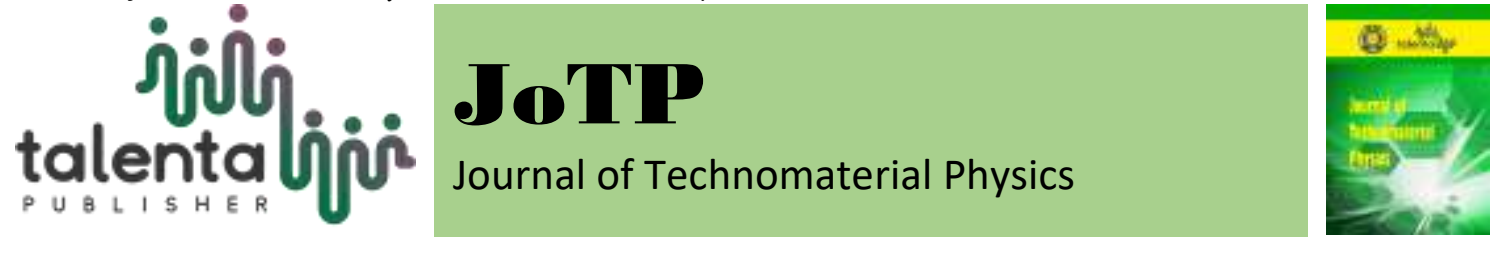

\title{
Theoretical Study of Negative Material Bias Index Symptoms
}

\author{
Tua Raja Simbolon ${ }^{1 *}$, Syahrul Humaidi ${ }^{2}$ and Adimas Agung ${ }^{3}$ \\ 1,2,3 Department of Physics, Faculty of Mathematics and Natural Science, Universitas Sumatera Utara \\ 20155, Indonesia
}

\begin{abstract}
Theoretical research on the phenomenon of negative refractive index material was cunducted. The material is a known metamaterial that can manipulate electromagnetic waves through it. By changing the permeability and permittivity of a medium that is worth imaginary, then the value is included in the equation will produce a refractive index that is negative. This value can be achieved through material forms are designed so that the magnetic field and electric field manipulation. The equation used on electromagnetic waves that Maxwell's equations, is revealed to be some similarities propagation magnetic field and electric field. The Methods Finite Difference Time Domain (FDT D) as a modifier equation was then equations analysis, simulated using MatlabR2014 program. Based on simulation results, visible when the electromagnetic waves through a material metamaterial, the waves are deflected, do not pass part of the material covered.
\end{abstract}

Keyword: negative refractive index, permeability, permittivity, FDTD

Received 20 October 2019 | Revised [08 November 2019] | Accepted [14 February 2020]

\section{Introduction}

Currently the role of technology is very important in people's lives. Technology is increasingly developing and experiencing changes, initially it was impossible, now it is possible, some of which have been proven. This development is inseparable from physical science which explains the phenomena that occur. One of the phenomena currently being developed is metamaterials, which are materials that can have a negative refractive index. [1-3].

In geometric optics, the refraction of light described by Snellius's Law occurs simultaneously with the reflection of these light waves, as explained by the Fresnel equation during the transition to the era of physical optics. The collision between the light wave and the interface of the two mediums causes the phase speed of the light wave to change. The wavelength will increase or decrease with the same frequency due to the nature of light waves which are transverse (not longitudinal). This knowledge led to the invention of the lens and refracting telescope. Refraction in the era of physical optics is described as a phenomenon of changing the

\footnotetext{
*Corresponding author at: Jl. Bioteknologi No.1 Kampus USU, Medan, Indonesia, 20155

E-mail address: tuaraja@usu.ac.id
} 
direction of wave propagation which does not only depend on changes in velocity, but also occurs due to other factors called diffraction and dispersion [4-6].

In 1968, Veselago said in his writing, what if the material had a negative refractive index. However, this paper is neglected, because at that time no material with a negative refractive index was found. At present, after the phenomenon of refraction is found in a material that has a negative refractive index, it will certainly provide opportunities for making perfect lenses and invisible cloaking (Harry Potter cloaks) which are very useful later [7].

\section{Methods and Scheme}

\subsection{Flow chart}

The research flow is given in the form of a flowchart as in Figure 1.

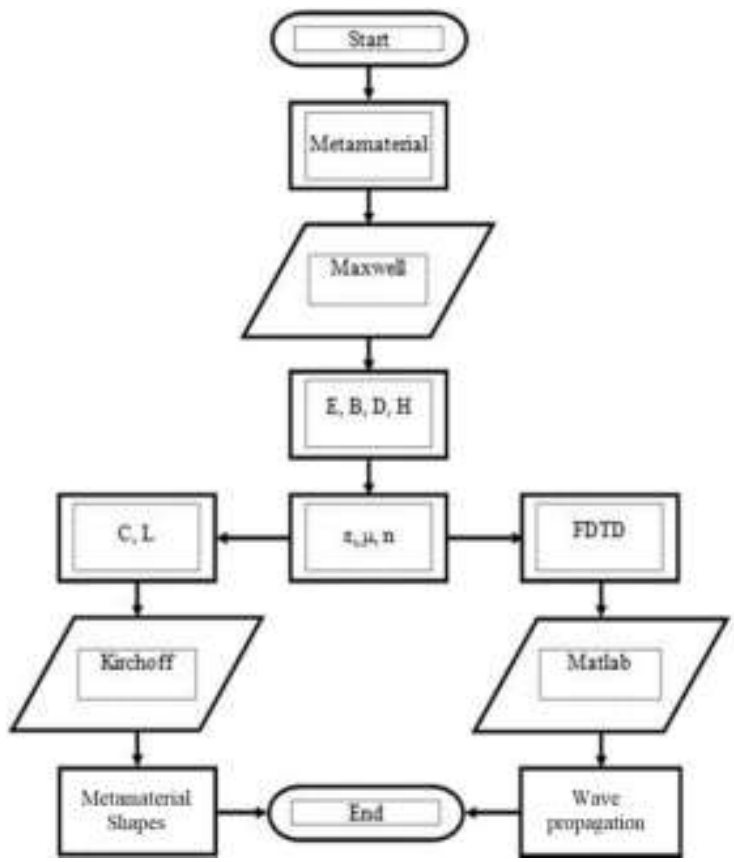

Figure 1. Research Flowchart

In Figure 1, the research flowchart begins with a start then the Metamaterial is studied through Maxwell's equations. Maxwell's equation will produce an electromagnetic wave equation, namely electric field, magnetic field, magnetic field density, and electric field density. Furthermore, by looking for general equations, a general solution will be obtained that will produce permittivity and permeability values which are then linked to the refractive index. After that this equation is studied in two major parts, first, it is solved in the form of an LC series by Kirchoff's law to obtain the form of a metamaterial. Second, by converting the equation into FDTD form which is then solved by the Matlab program, then it will produce a simulation of electromagnetic wave propagation. Then draw conclusions and end with end as the end of the study. 


\section{Result and Discussion}

\subsection{Material Negative Refractive Index}

Maxwell's equation in a vacuum can be written as follows:

$$
\begin{gathered}
\vec{\nabla} \cdot \vec{E}=0 \\
\vec{\nabla} \cdot \vec{B}=0 \\
\vec{\nabla} \times \vec{E}=-\frac{\partial \vec{B}}{\partial t} \\
\vec{\nabla} \times \vec{E}=\mu_{0} \epsilon_{0} \frac{\partial \vec{E}}{\partial t}
\end{gathered}
$$

To get a solution to the wave equation, the above equation can be converted into:

$$
\begin{gathered}
\vec{\nabla} \times \vec{E}=-j \omega \vec{B}=-j \omega \mu \vec{H} \\
\vec{\nabla} \times \vec{H}=j \omega \vec{D}+J=j \omega \varepsilon \vec{E}+J \\
\vec{\nabla} \cdot \vec{D}=\vec{\nabla} \cdot \varepsilon \vec{E}=\rho_{c} \\
\vec{\nabla} \cdot \vec{B}=\vec{\nabla} \cdot \mu \vec{H}=0
\end{gathered}
$$

In Ampere's law, when the current medium is freely flowing, then

$$
J=\sigma \vec{E}
$$

so that:

$$
\vec{\nabla} \times \vec{H}=j \omega \vec{D}+J=j \omega \varepsilon \vec{E}+\sigma \vec{E}=j \omega\left(\varepsilon+\frac{\sigma}{j \omega}\right)=j \omega\left(\varepsilon+\frac{j \sigma}{\omega}\right) \vec{E}
$$

This shows that in the phasor domain, conductivity can be related to permittivity to produce a new, effective complex permittivity:

$$
\varepsilon_{c}=\varepsilon+\frac{j \sigma}{\omega}=\varepsilon_{0} \varepsilon_{c r}=\varepsilon_{0}\left(\varepsilon_{r}^{\prime}-j \varepsilon^{\prime \prime} r\right)
$$

The equation can be simplified to:

$$
\vec{\nabla} \times \vec{H}=j \omega \varepsilon_{c} \vec{E}
$$

with $\varepsilon_{c}$ the complex number.

It was found that the permeability of the complex:

$$
\mu_{c}=\mu-\frac{j \sigma}{\omega}=\mu_{0} \mu_{c r}=\mu_{0}\left(\mu_{r}^{\prime}-j \mu^{\prime \prime}{ }_{c r}\right)
$$

Value theory, $\varepsilon_{0}=8.854 \times 10^{-12} \mathrm{~F} \cdot \mathrm{m}^{-1}$ and $\mu_{0}=4 \pi \times 10^{-7} \mathrm{H} \cdot \mathrm{m}^{-1}$ which is the permeability in a vacuum, while the imaginary part $\varepsilon_{\mathrm{r}}{ }^{\prime \prime}$ and $\mu_{\mathrm{r}}{ }^{\prime \prime}$ is the part related to the conductivity of the material, but the value must be positive in the medium to satisfy the conservative energy. 
With permittivity and permeability values

$$
\begin{gathered}
\sqrt{\varepsilon_{r}}=\sqrt{-\left|\varepsilon_{r}\right|}=j \sqrt{\varepsilon_{r}} \\
\sqrt{\mu_{r}}=\sqrt{-\left|\mu_{r}\right|}=j \sqrt{\mu_{r}}
\end{gathered}
$$

The wave number can be written as follows:

$$
k=\omega \sqrt{\varepsilon_{0} \varepsilon_{r}} \sqrt{\mu_{0} \mu_{r}}=-\omega \sqrt{\varepsilon_{0} \varepsilon_{r}} \sqrt{\mu_{0} \mu_{r}}
$$

Furthermore, it is connected with the refractive index equation that

$$
\begin{gathered}
n=\frac{c}{v} \\
n=\sqrt{\varepsilon_{r}} \sqrt{\mu_{r}}=-\sqrt{\left|\varepsilon_{r}\right|\left|\mu_{r}\right|}
\end{gathered}
$$

Table 1. Results $n$ of $\mu_{r}$ and $\varepsilon_{r}$

\begin{tabular}{ccc}
\hline$n$ & $\varepsilon_{r}>$ & $\varepsilon_{r}<$ \\
\hline$\mu_{r}>$ & + & $\mathrm{J}$ \\
$\mu_{r}<$ & $\mathrm{J}$ & - \\
\hline
\end{tabular}

Table 1 shows that a material can have a negative refractive index if its permeability and permittivity values are less than zero.

\subsection{Theory of Forms of Materials Having a Negative Refractive Index}

Materials that are negatively indexed have a permittivity and permeability value smaller than zero. Furthermore, the form of the material can be considered with the material composed of capacitors and inductors, as it is known that:

$$
C=\varepsilon_{0} \varepsilon_{c r} \frac{A}{d}
$$

While inductance can be written as:

$$
L=\frac{\mu_{0} N^{2} A}{l}
$$

Then the LC circuit can be used to produce a negative refractive index value with the following relationship:

$$
C \sim \varepsilon_{0} \operatorname{dan} L \sim \mu_{0}
$$

The current that flows in a circle results in the induction of a magnetic field that is time dependent. With phasors given in Figure 2 and Figure 3. 


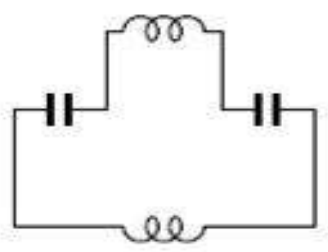

Figure 2. LC Circuit
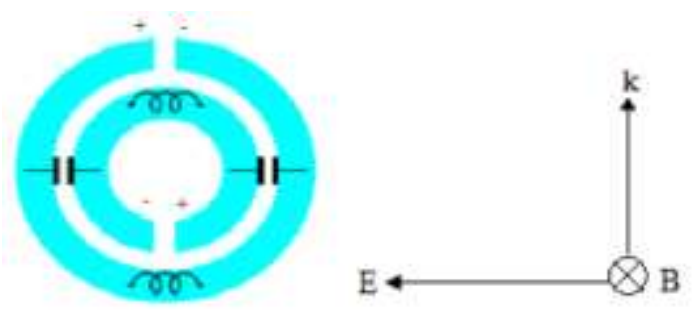

Figure 3. The direction of the electric field, magnetic field, and wave propagation in the split double ring

The above form can produce negative permeability and permeability values due to the wave motion that passes through it to meet the wave propagation phasor. Where is the value of the equation:

$$
\sqrt{\varepsilon_{r}}=\sqrt{-\left|\varepsilon_{r}\right|}=j \sqrt{\varepsilon_{r}} \sqrt{\mu_{r}}=\sqrt{-\left|\mu_{r}\right|}=j \sqrt{\mu_{r}}
$$

\subsection{Transmission of Electromagnetic Waves on Metamaterials}

From Faraday's law and Ampere's law that

$$
\begin{gathered}
\vec{\nabla} \times \vec{E}=-\frac{\partial \vec{B}}{\partial t} \\
\vec{\nabla} \times \vec{H}=\frac{\partial \vec{D}}{\partial t}
\end{gathered}
$$

This equation can be written as a wave equation that propagates in the z-axis direction as follows:

$$
\begin{aligned}
& \vec{H}_{y}^{n+\frac{1}{2}}\left[k+\frac{1}{2}\right]=\vec{H}_{y}^{n-\frac{1}{2}}\left[k+\frac{1}{2}\right]+\frac{\Delta t}{\mu \Delta z}\left[\vec{E}_{x}^{n}(k)-\vec{E}_{x}^{n}(k+1)\right] \\
& \vec{E}_{x}^{n+1}[k]=\vec{E}_{x}^{n}[k]+\frac{\Delta t}{\varepsilon \Delta z}\left[H_{y}^{n+\frac{1}{2}}\left(k-\frac{1}{2}\right)-\vec{H}_{y}^{n+\frac{1}{2}}\left(k+\frac{1}{2}\right)\right]
\end{aligned}
$$

In the FDTD algorithm, the components of the magnetic field magnitude are calculated from the electric field magnitudes. By using the magnetic field component that has been in the form of a 
new equation, it can be simulated [8-10]. For example, by updating the FDTD equation with the Drude Model method. From the electric flux density equation with the electric field.

That $\vec{D}=\sigma \vec{E}$. With $\varepsilon=\varepsilon_{r} \varepsilon_{0}$ and $\varepsilon_{r}$ in the Drude model given in the equation:

$$
\varepsilon_{r}(\omega)=\varepsilon_{\infty}-\frac{\omega_{p}^{2}}{\omega^{2}-j \gamma \omega}
$$

The substitution of the Drude model in the previous equation will be:

$$
\omega^{2} \vec{D}-\gamma(j \omega) \vec{D}=\varepsilon_{\infty} \omega^{2} \vec{E}-\omega_{p}^{2} \vec{E}-\varepsilon_{\infty} \gamma(j \omega) \vec{E}
$$

According to this equation, the value of the frequency function can be converted into a function of time with the relationship and. Also the product by is the average time. These two parts are used in either the first derivative or the second derivative for the conditions to be met. Finally, the form above can be written as:

$$
\begin{gathered}
\vec{E}_{x}^{n+1}=a_{e}\left(\vec{D}_{x}^{n+1}-2 \vec{D}_{x}^{n}+\vec{D}_{x}^{n-1}\right)+b_{e}\left(\vec{D}_{x}^{n+1}-\vec{D}_{x}^{n-1}\right)+c_{e}\left(2 \vec{E}_{x}^{n}-\vec{E}_{x}^{n-1}\right)+d_{e}\left(2 \vec{E}_{x}^{n}\right. \\
\left.+\vec{E}_{x}^{n-1}\right)+e_{e}\left(\vec{E}_{x}^{n-1}\right)
\end{gathered}
$$

The value of the additional variable in the new equation is scalar, namely:

$$
\begin{gathered}
a_{e}=\frac{4}{g} ; b_{e}=\frac{\gamma(2 \Delta t)}{g}, c_{e}=\frac{4 \varepsilon_{0} \varepsilon_{\infty}}{g}, d_{e}=\frac{\varepsilon_{0} \omega_{p}^{2}(\Delta t)^{2}}{g}, e_{e}=\frac{\varepsilon_{0} \varepsilon_{\infty} \gamma_{e}(2 \Delta t)^{2}}{g} \\
g=4 \varepsilon_{0} \varepsilon_{\infty}+\varepsilon_{0} \omega_{p}^{2}(\Delta t)^{2}+\varepsilon_{0} \varepsilon_{\infty} \gamma_{e}(2 \Delta t)
\end{gathered}
$$

In the same way additional variables will be obtained for the magnetic field equation.

$$
\begin{gathered}
\vec{H}_{y}^{n+1}=a_{m}\left(\vec{B}_{y}^{n+1}-2 \vec{B}_{y}^{n}+\vec{B}_{y}^{n-1}\right) b_{m}\left(\vec{B}_{y}^{n+1}-\vec{B}_{y}^{n-1}\right)+c_{m}\left(2 \vec{H}_{y}^{n}-\vec{H}_{y}^{n-1}\right) \\
d_{m}\left(2 \vec{H}_{y}^{n}+\vec{H}_{y}^{n-1}\right)+e_{m}\left(\vec{H}_{y}^{n-1}\right)
\end{gathered}
$$

Then the wave propagating in the $\mathrm{z}$ direction, the FDTD equation is as follows:

$$
\begin{aligned}
& \vec{B}_{x}^{n+1}(k)=\vec{B}_{x}^{n}+\frac{\Delta t}{\Delta z}\left(\vec{E}_{x}^{n}(k)-\vec{E}_{x}^{n}(k+1)\right. \\
& \vec{D}_{x}^{n+1}(k)=\vec{D}_{x}^{n}+\frac{\Delta t}{\Delta z}\left(\vec{H}_{y}^{n}(k)-\vec{H}_{y}^{n}(k+1)\right.
\end{aligned}
$$

The above equation provides the FDTD algorithm for $\vec{B}_{y}$ and $\vec{D}_{x}$.

The Refractive Index can be calculated from the equation:

$$
n_{F D T D}=\frac{1}{j k_{0}\left(z_{1}-z_{2}\right)} \log \left|\frac{\tilde{E}_{\chi}\left(\omega, z_{2}\right)}{\tilde{E}_{x}\left(\omega, z_{1}\right)}\right|
$$


where $\mathrm{k}_{0}$ is the regulated wave number on $\frac{\omega_{0}}{c}$ and the electric field is set at the location $z_{1}=$ $1415 \Delta z$ and $z_{2}=1424 \Delta z$

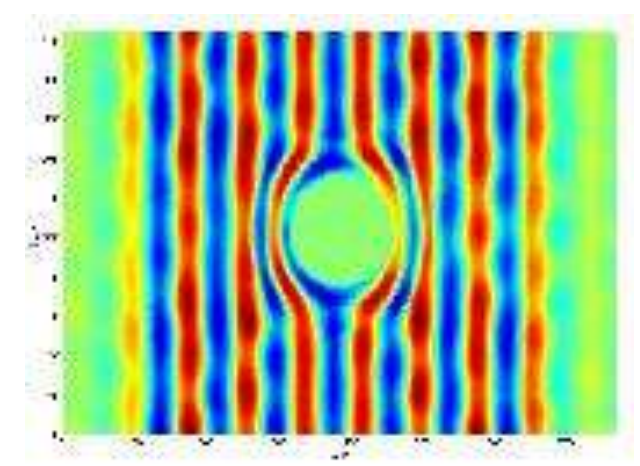

Figure 4. Simulation Results of Wave Spread on the Invisible Cloak (Cylinder)

\section{Conclusion}

1. After obtaining the equation of the real and imaginary parts of permit tivity and permeability, when connected with the refractive index, where the refractive index is the ratio of the speed of light in one medium to another, namely the equation $n=\frac{c}{v}$ dan $n=\sqrt{\varepsilon_{r}} \sqrt{\mu_{r}}=\sqrt{\left|\varepsilon_{r}\right|\left|\mu_{r}\right|}$ will produce a negative refractive index value. So that we get a material that has a negative refractive index value.

2. Inductors and capacitors have a relationship with the value of the permeability and permeability of a material. The equation is $L=\frac{\mu_{0} N^{2} A}{l}$ and $C=\varepsilon_{0} \varepsilon_{r} \frac{A}{d}$ so that by using the LC circuit, a form of metamerial material can be made, with the condition that the permittivity and permeability of the circuit are negative. This negative value can be generated by arranging a shape from the LC circuit according to the direction of the magnetic field and the electric field and the direction of propagation.

3. After obtaining a negative refractive index value, this phenomenon can be utilized in various fields such as for perfect lenses that exceed the Rayleigh Limit so that a disc (DVD) has a very large capacity, and an invisible cloak that can create something when covered by it will not be seen. This is in accordance with the simulation results on wave propagation for the cylinder, which is clear that the GEM cannot enter (penetrate) into the cylinder but instead the cylinder deflects the GEM that passes through it so that it is said that the cylinder can manipulate the GEM that propagates around it, in the end what is inside the cylinder or cylinder itself, not visible from the outside. 


\section{Acknowledgement}

To the staff of Laboratorium Instrumentasi Digital and Mrs. Dr. Wika Hanida Lubis, Mked(PD), SpPD-Kpsi, FINASIM, Niken R Damanik, S.Ked for the guidance in understanding human stress physiology.

\section{REFERENCES}

[1] T. J. Cui, D. Smith and R. Liu, Metamaterials: Theory, Design, and Applications, New York: Springer, 2010.

[2] A. C. Nicolaide, Electromagnetics: General Theory of the Electromagnetic Field, Romania: Transilvania University Press, 2002.

[3] N. Engheta and R. W. Ziolkowski, Metamaterials: Physics and Engineering Explorations, New York: Wiley-IEEE Press, 2006.

[4] M. Born and E. Wolf, Principles of Optics: Electromagnetic Theory of Propagation, Interference and Diffraction of Light 7th Edition, England: Cambridge University Press, 2013.

[5] A. S. Vioktalamo, R. Watanabe and T. Ishihara, "Permeability Enhancement of Stratified Metal Dielectric Metamaterial in Optical Regime," Photonics and NanostructuresFundamentals and Applications, vol. 10, no. 3, pp. 325-328, 2012.

[6] M. Notomi, "Theory of Light Propagation in Strongly Modulated Photonic Crystals: Refractionlike Behavior in the Vicinity of the Photonic Band Gap," Phys. Rev. B, vol. 62, no. 16, pp. 10696-10705, 2000.

[7] W. J. Padilla, D. N. Basov and D. R. Smith, "Negative Refractive Index Metamaterials," Materialstoday, vol. 9, no. 7-8, pp. 28-35, 2006.

[8] M. Fusco, "FDTD Algorithm in Curvilinear Coordinates (EM Scattering)," in Proceeding 1990. IEEE Transactions on Antennas and Propagation, vol. 38 no. 1, pp. 76-89, 1990.

[9] T. Namiki, "A New FDTD Algorithm Based on Alternating-Direction Implicit Method," in Proceeding IEEE Transactions on Microwave Theory and Techniques, vol. 47, no. 10, pp. 2003-2007, 1999.

[10] J. Shibayama, M. Muraki, J. Yamauchi and H. Nakano, "Efficient Implicit FDTD Algorithm Based on Locally One Dimensional Scheme," Electronic Letters, vol. 41 no. 19, pp. 1046-1047, 2005. 